\title{
Recent Biotechnological Applications Using Oleosins
}

\author{
N.J. Roberts ${ }^{1}$, R.W. Scott ${ }^{1}$ and J.T.C. Tzen ${ }^{2,3, *}$ \\ ${ }^{1}$ Forage Biotechnology, AgResearch Ltd., Private Bag 11008, Tennent Drive, Palmerston North, New Zealand, 4442; \\ ${ }^{2}$ Graduate Institute of Agricultural Biotechnology, National Chung-Hsing University, Taichung, Taiwan 40227, ROC \\ and ${ }^{3}$ Agricultural Biotechnology Research Center, Academia Sinica, Taipei 115, Taiwan
}

\begin{abstract}
Oleosins are naturally occurring, small (15-24 kDa), amphipathic, plant proteins that prevent the coalescence of oil bodies (OBs) during seed and pollen maturation. The physiochemical properties of oleosins and their association with OBs have led to a broad array of potential applications in biotechnology utilizing native or recombinant forms of oleosin or oleosin-fused polypeptides. This review begins by briefly outlining the current understanding of oleosin topology, oil body assembly and potential health issues. A schematic model is given to potentially explain the apparent simultaneous existence of parallel and anti-parallel $\beta$ sheets and a figure summarizing the process of oleosin translation through to oil body formation in vivo is also presented. The applications for oleosins, the associated modes of action and their relevant patents are then discussed in six areas: recombinant protein purification; generating protein complexes; in planta delivery; emulsification; artificial oil bodies; and modifications to the properties of oleosin itself by creating polyoleosin.
\end{abstract}

\section{INTRODUCTION}

Flowering plants store triacylglycerol (TAG) in their seeds as an energy source for germination. The TAG is contained within discreet structures called oil bodies (OBs), which are $0.5-2 \mu \mathrm{m}$ in diameter and consist of a TAG core surrounded by a phospholipid monolayer embedded with proteinaceous emulsifiers - predominantly oleosins [1]. OBs consist of $0.5-3.5 \%$ protein; of this $80-90 \%$ is oleosin with the majority of the remainder consisting of the proteins caleosin (calcium binding) and steroleosin (sterol binding) [2]. The role of the oleosins is to stop the OBs coalescing as the cells dehydrate; thus maintaining the appropriate surface area/volume ratio of each $\mathrm{OB}$ and ensuring the rapid availability of TAG during germination. Oleosins have also been reported in pollen [3-5] and the female gametophyte of gymnosperms [6].

The unique properties of oleosins form the basis of a number of applications including: purifying proteins; formation of multimeric protein complexes; emulsification; delivery of bioactives; generation of multivalent bioactives and even as a potential flavour enhancer. In order to more fully understand the principals of these applications it is necessary to understand the properties of oleosins; it is the purpose of this review to discuss their properties and applications.

\subsection{Oleosin Topology}

Oleosins have three structural domains consisting of an amphipathic N-terminal domain, a central hydrophobic domain and a C-terminal amphipathic domain. They are comparatively small proteins with a molecular mass ranging from 15 to $24 \mathrm{kDa}$ where the variation is predominantly due to different lengths of the $\mathrm{N}$ - and $\mathrm{C}$-terminal domains. The accepted topological model is one in which the $\mathrm{N}$ - and

*Address correspondence to this author at the Graduate Institute of Biotechnology, National Chung-Hsing University, Taichung, Taiwan; Tel: 886-422840328; Fax: 886-4-22853527; E mail: TCTZEN@dragon.nchu.edu.tw
C-terminal amphipathic domains are located on the outside of the OBs and the central hydrophobic domain is located inside the OB (Figs. 1A-F and 2) [7-9]. The negatively charged residues of the $\mathrm{N}$ - and C-terminal amphipathic domains are exposed to the aqueous exterior whereas the positively charged residues are exposed to the $\mathrm{OB}$ interior and face the negatively charged lipids. Thus, the amphipathic domains with their outward facing negative charge are responsible for maintaining the OBs as individual entities via steric hinderance and electrostatic repulsion both in vivo and in isolated preparation [1]. The N-terminal amphipathic domain is highly variable and as such no specific secondary structure can describe all examples, in comparison the Cterminal domain contains an $\alpha$-helical domain of 30-40 residues [2].

The central domain is highly conserved and thought to be the longest ( $\sim 72$ residues) hydrophobic domain known to occur in nature; at the center is a conserved 12 residue proline knot motif which includes three spaced proline residues (for reviews see $[2,10]$ ). The secondary, tertiary and quaternary structure of the central domain is still unclear. Modelling, Fourier Transformation-Infra Red (FT-IR) and Circular Dichromism (CD) evidence exists for a number of different arrangements. Tzen et al., [1] proposed the hydrophobic domain exists as anti-parallel $\beta$ strands with the proline knot deeply embedded in the TAG core (Fig. 1A); this was further suggested [2] to fold into a 6 stranded antiparallel $\beta$-barrel (Fig. 1B). Li et al. [11,12] put forward an intermolecular $\beta$-sheet structure which extends around the surface of the $\mathrm{OB}$ and is formed by the parallel arrangement of separate oleosin strands (Fig. 1C). The formation of natural homo oligomers seen when purifying oil bodies even with strong denaturants (such as SDS) was used to support the proposed natural formation of hydrogen bonds between adjacent oleosin molecules [12]. Two additional tertiary and quaternary arrangements can be extrapolated from the intermolecular $\beta$-sheet structure, i.e. anti-parallel $\beta$ sheets (Fig. 1D) and a mixture of parallel and anti-parallel $\beta$ sheets (Fig. 
1E). In comparison, Lacey et al. [13] and Alexander et al. [14] proposed the hydrophobic domain consists of two $\alpha$ helices with the proline knot motif forming a $180^{\circ}$ turn; the whole structure was thought to be monomeric and stabilised by internal H-bonds with no $\beta$-sheet (Fig. 1F). All arrangements take account of the potential flexibility offered by conserved tandem glycine or sequential glycine-serine residues, as well as residue pairing enabling intramolecular and/or intermolecular hydrogen bond formation. Given the nature and length of the hydrophobic domain as well as the plasticity of its surrounding environment, it is possible that a single OB can simultaneously contain more than one of these forms.

\subsection{OB Assembly}

OB formation begins with the accumulation of TAGs between the layers of the ER lipid bilayer membrane; the enlarging TAG droplet and surrounding phospholipid monolayer (derived from the outer layer of the ER membrane) is eventually pinched off (Fig. 2). Prior to this separation, the surrounding phospholipid monolayer is embedded with oleosins and it is the ratio of oleosin/TAG that determines the size of the OBs $[15,16]$. However, the separation of the OB from the ER may not be the final process in its maturation since OBs formed at the beginning of seed development were comparatively deficient in oleosin compared to OBs analysed at the end of seed maturation, indicating that the OBs either coalesce to reach a stable oleosin:TAG ratio [16] or they re-fuse with the ER membrane prior to eventual maturation (for review see [17]) (Fig. 2).

Recombinant oleosins were shown to be targeted to oil bodies in transformed yeast [18]; building on this, Beaudoin et al. [19] used a series of yeast secretory mutants to demonstrate that the process requires the signal recognition particle

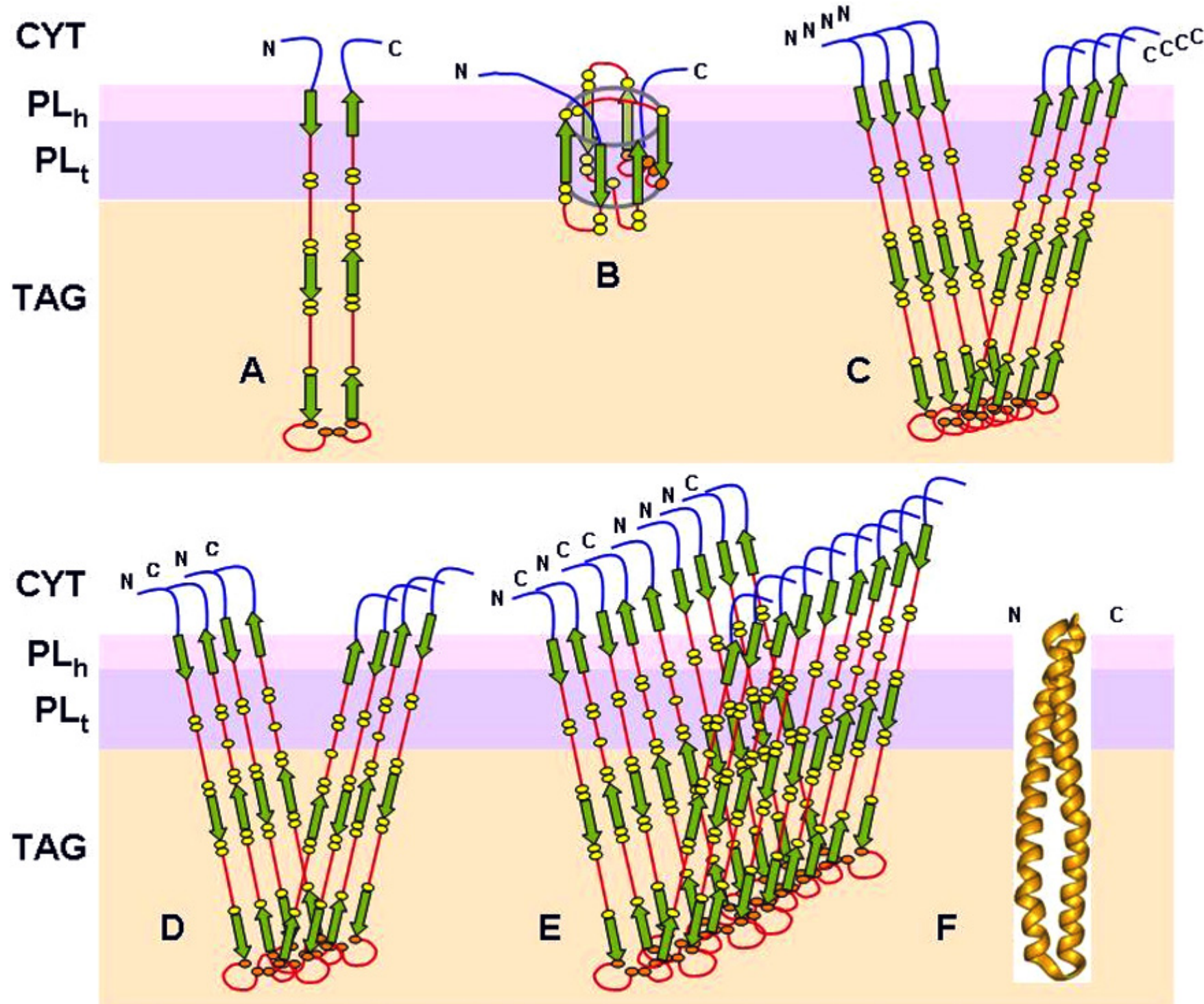

Fig. (1). Potential secondary, tertiary and quaternary structures of the hydrophobic central domain (not drawn to scale). A) monomeric, antiparallel $\beta$ strands/sheet hairpin; B) anti-parallel $\beta$ barrel; C) oligomeric parallel $\beta$ sheet; D) oligomeric anti-parallel $\beta$ sheet; E) oligomeric mixed parallel/anti-parallel $\beta$ sheet; F) $\alpha$ helix hairpin [figure from Capuano et al., 2007, used with kind permission from Professor Peter Shewry]. $\beta$ strands are shown as green arrows, invariant residues in the proline knot are shown as orange circles and semi conserved glycine and serine residues in the flexible portions are shown as yellow circles. CYT $=$ cytoplasm, $\mathrm{PL}_{\mathrm{h}}=$ phospholipid head, $\mathrm{PL}_{\mathrm{t}}=$ phospholipid tail, $\mathrm{TAG}=$ triacylglycerol. 


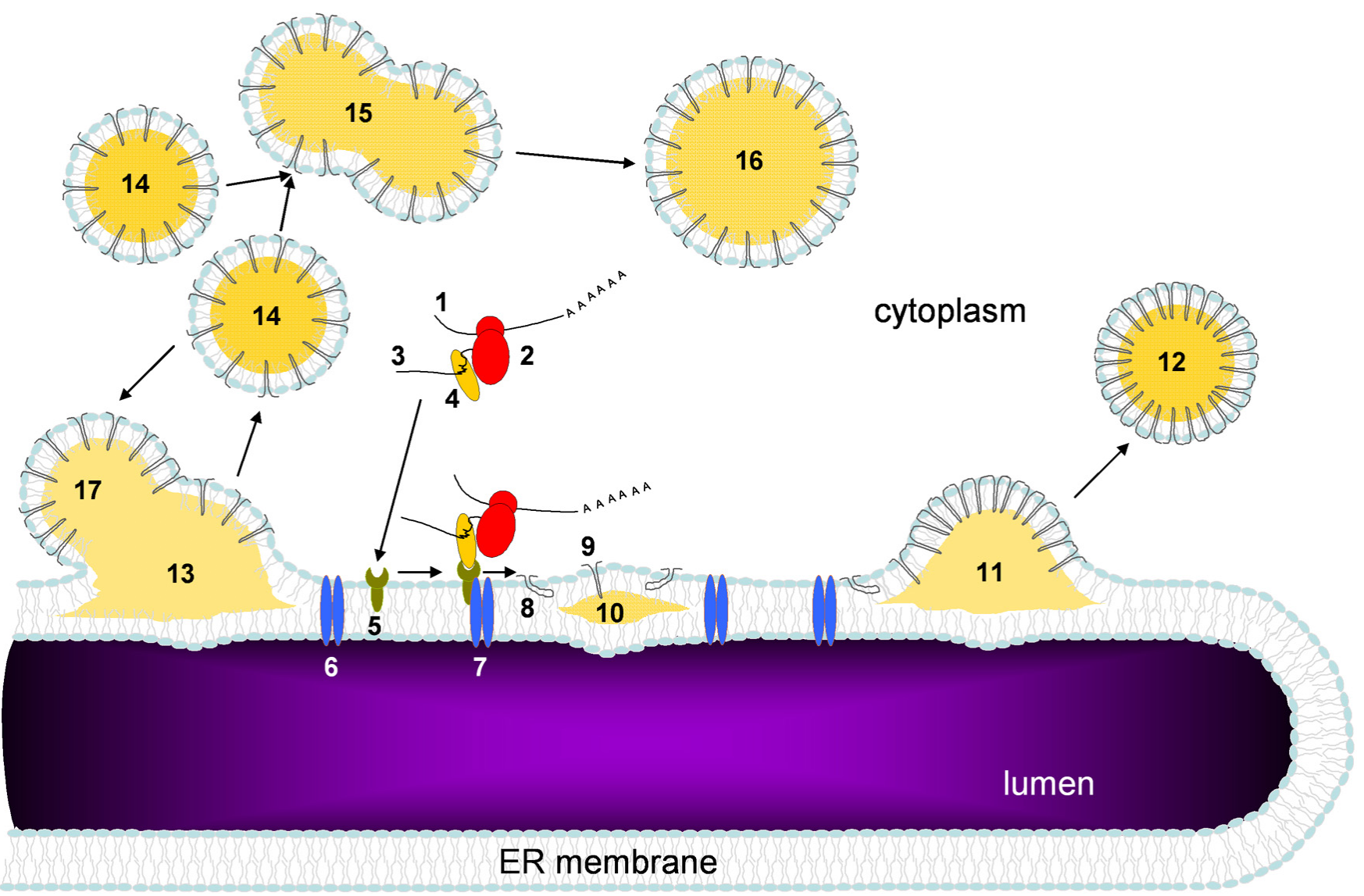

Fig. (2). Schematic representation of oil body assembly (modified from [16,19,23]). Phospholipids are shown with light blue polar heads and grey lipid tails, oleosins are shown as dark grey, triacylglycerol (TAG) shown as light orange. 1) oleosin mRNA; 2) ribosome; 3) nascent oleosin peptide with unidentified signal sequence; 4) signal recognition particle (SRP); 5) SRP receptor; 6) translocation - insertion site; 7) oleosin translation machinery/SRP/translocation - insertion site complex; 8) constrained oleosin in ER membrane; 9) relaxed oleosin in oil body (OB) phospholipid monolayer - TAG core; 10) initial accumulation of TAG; 11) maturing OB with maximum density of oleosins; 12) OB with maximum density of oleosins released from the ER membrane 13) maturing OB with low density of oleosins; 14) OB with low density of oleosins released from the ER membrane; 15) coalescing OBs with low density of oleosins; 16) expanded OB with increased density of oleosins; 17) OB with low density of oleosins coalescing with maturing OB also with low density of oleosins.

(SRP) for targeting to the ER as well as a functional SEC61 translocon for correct insertion into the membrane. It is thought that direct targeting of the central hydrophobic domain to the maturing oil bodies occurs at the point where the ER membranes are enlarged with TAG molecules (Fig. 2). Similarly, this applies to the caleosins, which like oleosins, also have a freely exposed N-terminus and oil body anchoring structure [20]. While no specific targeting sequence has been identified, the poor accumulation of oleosins in OBs after insertion of the oleosin N-terminus into the lumen of the ER (via a fused signal sequence) or disruption of the oleosin proline knot motif indicates the importance of these regions in this process [21]. Sarmiento et al., [22] and Abell et al. [23] suggest that in the TAG environment oleosin adopts a more favourable conformation than in the ER membrane and this could be a thermodynamic driver for oleosin targeting to regions of TAG formation (Fig. 2). Interestingly, the exact sequences of the hydrophobic regions flanking the proline knot motif appear to tolerate a high degree of variation and yet still targets to (or accumulates on) the OB [23]. In contrast, steroleosin possesses a non-cleavable signal sequence and is also thought to target to the ER via the SRP dependent pathway and is anchored to the oil bodies primarily via its N-terminal hydrophobic domain [24]. After depo- sition into the OB membrane, oleosins are thought to have the potential to form oligomers via hydrogen bonds between their central hydrophobic domains [12].

The absence of oleosin does not prevent the formation of OBs but rather results in a large variation in the size and stability of the OBs with the average size increasing dramatically and correlating with a decline in stability [16]. Conversely, the normal spherical shape of the OBs can be severely altered by raising the ratio of oleosin:TAG, essentially resulting in the formation of incompletely filled OBs which are irregularly shaped [15]. The stability of OBs can be substantially enhanced after their surface proteins are crosslinked by genipin [25].

\subsection{Possible Health Concerns}

The proposed uses of oleosin as an emulsifier or delivery tool for fused proteins or lipid soluble drugs either into the circulatory stream or digestive tract and possibly even topical applications raises some potential health concerns. More specifically, there have been three relatively recent publications indicating that food allergies to peanut, hazelnut and sesame seed could in some part be due to the native oleosins contained in these edible plant organs [26,27,28 respec- 
tively]. ELISA, IgE-RIA and immunoblots were used to detect oleosin specific immunoglobulin (Ig)Es from a variety of patients displaying convincing allergic symptoms after ingestion of the plant organ or plant products, suggesting that oleosins can indeed act as allergens. In many cases prick tests failed to show evidence of specific IgEs to the oleosins, the possibility of specific IgEs recognising only relatively protected epitopes that are located inside the OBs was not supported by the ELISA results [28], furthermore the hydrophobic portion of oleosin located inside the OBs was reported to have induced no or nondetectable antibodies [26]. The frequency, specificity and severity of these allergic reactions is unknown and requires considerably more research.

\section{OLEOSIN APPLICATIONS}

The majority of the patented oleosin applications are summarised in Table 1. While Table $\mathbf{1}$ may not contain the latest updates - the theme, applications and inventors remain the same. The applications are discussed in more detail later in this article.

\subsection{Use of Oleosin for Purification}

Perhaps the most well-known commercial use for oleosin is as a carrier of high value recombinant peptides. The original patent [29] was based on the following three applications:

- Fusion of a recombinant protein to oleosin and expression in planta made it possible to use a very simple and efficient post harvest purification system.

- Fusion of a recombinant protein to oleosin and expression in planta allowed the potential to protect the seed and germinating seedling from pathogens.
- The persistent association of $\mathrm{OB}$ proteins with the $\mathrm{OB}$ enabled the immobilization of active enzymes on the outside of the $\mathrm{OB}$.

Since the initial filing of the patent, the system and associated patent has undergone numerous continuations as new proteins were tested; additional in planta applications were found, or modifications were made to the purification scheme; the latest patent filed being Moloney and van Rooijen [30]. Maurice Moloney, the original inventor of the system that utilized oleosin as a carrier, has successfully built a biotech company (SemBioSys) based on manufacturing high value recombinant proteins fused to oleosins and the use of non-recombinant oleosins for carriers of high value ingredients for personal topical applications and pharmaceutical applications.

\subsubsection{Principals of Using Oleosin::Recombinant Protein Fusions for Purification}

Purification of a refined product from any biological expression system comes at a cost and this process alone can determine commercial success or failure. The application of oleosin for recombinant protein purification is specifically aimed at lowering both capital costs for facilities and purification costs for each batch.

The system is based on the fact that in appropriately transformed plants the oleosin::recombinant protein fusions are targeted to the naturally occurring OBs in the seed. Following transgenic seed production, the harvested seed is milled in a suitable aqueous buffer and the OBs are purified from seed-derived impurities through a series of simple centrifugation and wash/elution steps changing salts and $\mathrm{pH}$, as in this aqueous environment OBs can be made to float. The single oleosin::recombinant protein fusion site is engineered

Table 1. Patents Involving Oleosins and their Applications

\begin{tabular}{|c|c|c|c|}
\hline Patent Title & Patent Number & Authors & Year \\
\hline Oil-body proteins as carriers of high-value peptides in plants & US $5,650,554$ & Moloney & 1997 \\
\hline Xylanase obtained from an anaerobic fungus & US $6,137,032$ & Cheng et al., & 2000 \\
\hline Oil body based personal care products & US $6,183,762$ B1 & Deckers et al., & 2001 \\
\hline Use of oil bodies & US $6,210,742$ & Deckers et al., & 2001 \\
\hline Oleosin/Phospholipid Complex and process for producing this same & WO 02/26788 A1 & Harada et al., & 2001 \\
\hline Products for topical applications comprising oil bodies & $\begin{array}{c}\text { US } 6,596,287 \text { B2 \& } \\
6,582,710 \text { B2 }\end{array}$ & Deckers et al., & 2003 \\
\hline Oil bodies and associated proteins as affinity matrices & US $6,509,453$ & Moloney et al., & 2003 \\
\hline Ice confection and its manufacturing process & US 2005/0037111A1 & Berry et al., & 2005 \\
\hline Recombinant oleosins from cacao and their use as flavouring or emusifying agents & US 7,126,042 B1 & McCarthy & 2006 \\
\hline Expression of epidermal growth factor in plant seeds & US 7,091,401, B2 & Moloney and Van Rooijen & 2006 \\
\hline Methods for the production of multimeric protein complexes, and related compositions & US 2006/0179514 A1 & Van Rooijen et al., & 2006 \\
\hline Methods for the production of multimeric immunoglobulins, and related compostions & US 7,098,383 & Szarka et al., & 2006 \\
\hline Polyoleosins & WO 2007045019 & Scott et al., & 2006 \\
\hline Stability enhancement of native and artificial oil bodies by genipin crosslink & I 250466 & Peng et al., & 2006 \\
\hline
\end{tabular}


to contain a unique cleavage site, enabling the recombinant protein to be separated from the oleosin into the aqueous buffer, while the oleosin remains embedded in the OBs. OBs containing the cleaved oleosin are removed by centrifugation while the cleaved recombinant protein can be further purified using conventional downstream processing. A later version of the patent [31] describes a system whereby the oleosin remains fused to a recombinant enzyme, and is used as means of recycling recombinant enzymes (xylanase, chitinase).

While oleosin can theoretically be fused to either the Nor C-terminus (or potentially even to both) this is not suitable for all proteins as it effectively targets the recombinant protein to the cytoplasm thereby limiting the exposure to various folding chaperones and post translational modifications. In addition, the costs of cleavage (e.g., enzyme cost, licensing, clean up) may be prohibitive.

Recombinant oleosin::recombinant protein fusions also have exploitable emulsification properties, for example oleosin::carp growth hormone fusion was used to generate a film forming emulsion which allowed it to be used as a stable coating of fish food and also created a timed release formulation [32].

\subsubsection{Principals of Using Oleosin::Ligands and $O B:$ : Ligands as Affinity Matrices for Purification/Removal}

A slight departure from the oleosin::protein fusion for protein purification is the oleosin::ligand fusion or OB::ligand for purification [33,34]. These patents demonstrate that it is possible to use oleosin::high affinity ligand fusions (such as Protein A) on OBs to purify ligand binding targets such as $\mathrm{IgG}$ antibodies (which bind to Protein A). The ligand can also be associated with OBs (not necessarily only oleosin) via its own natural affinity (e.g., an anti-OB antibody which is fused to a second ligand for which the target protein has an affinity). Purifying the desired ligand binding target is again based on the fundamental principal that in an aqueous environment OBs can be made to float. Thus, purification uses a similar approach to the oleosin::fusion procedure with milling, centrifugation and washing steps. Disruption of the ligand/target binding (e.g., by the addition of salts or changing $\mathrm{pH}$ ) allows for partitioning of the target to the aqueous phase which can be further purified using conventional downstream processing.

Some examples of ligands and their respective targets were hirudin/thrombin, metallothionein/cadmium, cellulose binding protein/cellulose, and protein A/immunoglobulins. In some cases this method avoids the need to make oleosin fusions to the desired target recombinant protein and therefore enables the recombinant protein to be targeted to the SRP dependent pathway for appropriate postranslational modification and/or assembly (required for immunoglobulins) prior to purification.

\subsection{Use of Oleosin for Generating Protein Complexes}

\subsubsection{Principals of Using Oleosin::Recombinant Protein Fusions for Generating Protein Complexes}

The patent by van Rooijen et al. [35] is a further variation on both the oleosin::recombinant protein fusion for purification and oleosin-ligands as affinity matrices. It relates to generating multimeric protein complexes, redox proteins and recombinant polypeptides. In one example, two proteins were fused individually to oleosin and a complex was formed when both proteins were brought into close proximity on the outside of the OB (e.g., thioredoxin and thioredoxin-reductase). In a second example, the first recombinant polypeptide was an immunoglobulin light chain (or active fraction), and the second recombinant polypeptide was an immunoglobulin heavy chain (or active fraction thereof).

\subsection{In Planta Delivery of Oleosin:Protein Fusions}

Moloney [31] detailed several oleosin::recombinant protein fusions which were designed to be delivered in planta. One of these was oleosin::tryptophan decarboxylase, which was shown to theoretically modify the seed secondary metabolites by depleting the tryptophan derived glucosinolates in the seed. Two other examples, oleosin::trypsin inhibitor and oleosin::chitinase, were also designed to be delivered in planta for protection from predation. However, these were also designed to be cleaved from oleosin via a second recombinant protein; for example, a recombinant enzyme such as collagenase expressed under the isocitrate lysase promoter, where the promoter is normally active during early stages of seed germination, but not in the developing seed. In this case the trypsin inhibitor or chitinase enzymes would be fused to oleosin via a linker that includes a collagenase recognition site, and released into the cytoplasm during seed germination.

Cheng et al. [36] describes the fusion of oleosin to a xylanase gene from the anaerobic fungus Neocallimastix patriciarum. This is intended for expression in canola where the breakdown of OBs during the canola oil extraction process releases oleosin::xylanase, and subsequently active xylanase. Xylanase activity promotes the breakdown of the prevalent xylan hemicellulose thus making more carbohydrate content readily available to the microorganisms in the rumen. This adds value to the remaining canola meal, which is used as an animal feed supplement.

\subsection{Use of Oleosin for Emulsification}

\subsubsection{Non-Transgenic Oleosin as an Emulsifier and Fla- vouring Agent}

Emulsions are produced when one or more liquids that are immiscible in another liquid, usually due to different polarities and thus different hydrophobicities, are uniformly suspended within that liquid. Examples include oil droplets uniformly dispersed in water, or water droplets uniformly dispersed in oil. Generation of a relatively stable emulsion requires the use of an emulsifier, which lowers the interfacial tension between the liquids. The stability of an emulsion is generally measured in terms of the duration that the uniform dispersion persists under specified conditions. Emulsifiers are commonly used in the food and cosmetic industry and thus should have high emulsion stability for the safety of consumption and topical application.

Intact OBs containing oleosin naturally form a surfactantfree, oil-in-water emulsion. It has been found that intact OBs or OBs in which the majority of TAG has been removed have a broad range of emulsification applications in food, topical personal care (skin creams) and pharmaceutical formulations $[32,37,38]$. These patents stress the necessity of removing contaminating, non-OB proteins to reduce allergenicity, odour, colour, and to increase stability. As noted in 
Section 1.3; however, oleosin itself may be allergenic. Depending on the source of the OBs and the desired final application, OB preparation was achieved through the careful extraction of intact OBs via imbibing, heating and specialised milling, followed by decantation centrifugation and washing the OB preparations with salts, sodium carbonate, $\mathrm{pH}$ changes, organic solvents and detergents (anionic and non ionic). Purified OBs were stable for up to two hours at $100^{\circ} \mathrm{C}$ and were resistant to relatively harsh chemical and physical conditions. Additional benefits to generating relatively pure OB preparations included the superior water and oil absorption characteristics, up to approximately $50 \%$ and $30 \%$, respectively [32]. Both Harada et al. [38] and Deckers et al. [32] describe the generation of formulations for food products such as mayonnaises. The patents also list a number of applications for personal care products - including moisturizers, emollients, fragrances and active ingredients attached either covalently or non-covalently to the OBs (e.g., sunscreen actives, anti-wrinkle and anti-aging, sun tanning actives, anti-microbial, anti-acne actives, anti psoriasis actives, anti eczema actives, topical anaesthetic actives, anti inflammatory actives, vitamin actives and protein actives).

Harada et al. [38], also showed that purified intact OBs from a wide variety of plants could be dried, this eliminated approximately $70 \%$ of the oil and enriched the oleosin/phospholipid fraction to the point where the oleosin was $>95 \%$ of the total protein fraction. The oleosin/phospholipid complex was then able to be used alone as an emulsion stabiliser, or combined with other emulsifiers and emulsion stabilisers. In contrast, Deckers et al. [32,39] appeared to have only worked with predominantly intact OBs for emulsification.

More details of formulations containing OBs as emulsifiers to generate ice confection and as a flavouring agent were detailed in Berry et al. [40] and McCarthy [41], respectively. The use of OBs to generate ice confection [40] was based on the fact that the $\mathrm{OB}$ is pre-emulsified, which not only reduced manufacturing costs but could also be used to deliver potentially health promoting polyunsaturated fats and vitamins incorporated into the OBs by the plant producing the OBs. The use of intact OBs reduced or eliminated the need to include milk solids not fat (MSNF; e.g., proteins), and meant the products should be cheaper to manufacture. However, it was reported that in order to maintain the integrity of the OBs the process of screening potential aerating agents needed to be modified. Interestingly, Deckers et al. [32] reported that slow freezing and subsequent thawing of washed oil body preparations disrupted the homogeneous emulsion. This could be prevented by flash freezing in liquid nitrogen or by adding glycerol to the oil body preparation prior to freezing. No such problems were reported by either Berry et al. [40] or McCarthy [41], suggesting that it may only occur with relatively purified washed oil bodies rather than in formulations. McCarthy [41] also hypothesised that raising oleosins in cacao seeds would provide a mechanism to increase the favour of cacao by generating more hydrophilic peptides and hydrophobic amino acids, the precursors to key cacao flavour components.

\subsection{Generation of Artificial Oil Bodies (AOBs) with Na- tive and Recombinant Oleosin}

Prokaryotically expressed recombinant oleosins can be used to generate artificial oil bodies (AOBs) [42]. The proc- ess of generating AOBs involves combining TAG, and the recombinant oleosin or oleosin::recombinant protein fusion contained within the insoluble inclusion bodies. In the absence of strong chaotropic agents the disruptive force required to dissociate the oleosin pellet involves several alternating cycles of sonicating and cooling.

Capuano et al. [43] speculated that oleosin in AOBs would have different topologies than oleosins in plant produced OBs. However, Peng et al. [44] compared native OBs with AOBs and found they behaved in a similar fashion, in terms of the ratio between oleosin and TAG in determining size distribution, thermo-stability, and structural stability. Furthermore, the anchorage of the oleosins was also similar, with purified prokaryotically expressed recombinant oleosins able to be inserted into oil and phospholipid monolayers, where they rapidly reduced the interfacial tension [45].

AOBs have been used in the same manner as native OBs for purifying recombinant proteins fused to the oleosin (Fig. 3); some examples include:

- GFP fused via a sequence susceptible to factor Xa cleavage [42].

- cystatin (a thermostable cysteine protease inhibitor) fused via a sequence susceptible to cleavage by papain [46].

- nattokinase (a serine protease with fibrinolytic activity) and nattokinase with a propeptide sequences fused by a self splicing intein fragment [47].

- hydatoinase (HDTar; an industrially important enzyme for generating intermediates in the production of antibiotics, peptide hormones, pyrethroids and pesticides) fusion to oleosin-intein resulted in a one step process to obtain the protein from an insoluble form and after self splicing yielded greater than a 300 fold purification of HDTar. It was also reported that the source of TAG used to generate the AOBs was found to be critical with the best results being obtained using canola oil or olive oil compared to soybean oil, vegetable oil, sunflower oil or mineral oil [48].

Since most applications for oleosins relies on their association with OBs, testing the potential of modified oleosins (e.g., fusions, deletions, and substitutions) in transgenic plant systems requires considerable time. The use of AOBs to analyse prokaryotically expressed modified oleosins offers substantial savings in time. A further advantage of using AOBs is that they do not contain wild type oleosin.

\subsection{Polyoleosin}

Polyoleosin is a series of homo- or hetero- multimeric oleosin tandem repeats. Joining multiple oleosin units head to tail alters the emulsification properties of oleosin as well as $\mathrm{OB}$ and AOB stability [49]. Specific amino acid residues between the oleosin repeats were added to allow sufficient flex between the repeats and provide convenient sub-cloning sites for the insertion of other in-frame recombinant peptide sequences. The exploitable benefits include:

- extending the thermal stability of emulsifications, thereby broadening their application in reconstituted feeds, pharmaceuticals, beauty products, topical applications, etc.

- altering the number of oleosin repeats allows for the controlled release of lipid soluble bioactive compounds 

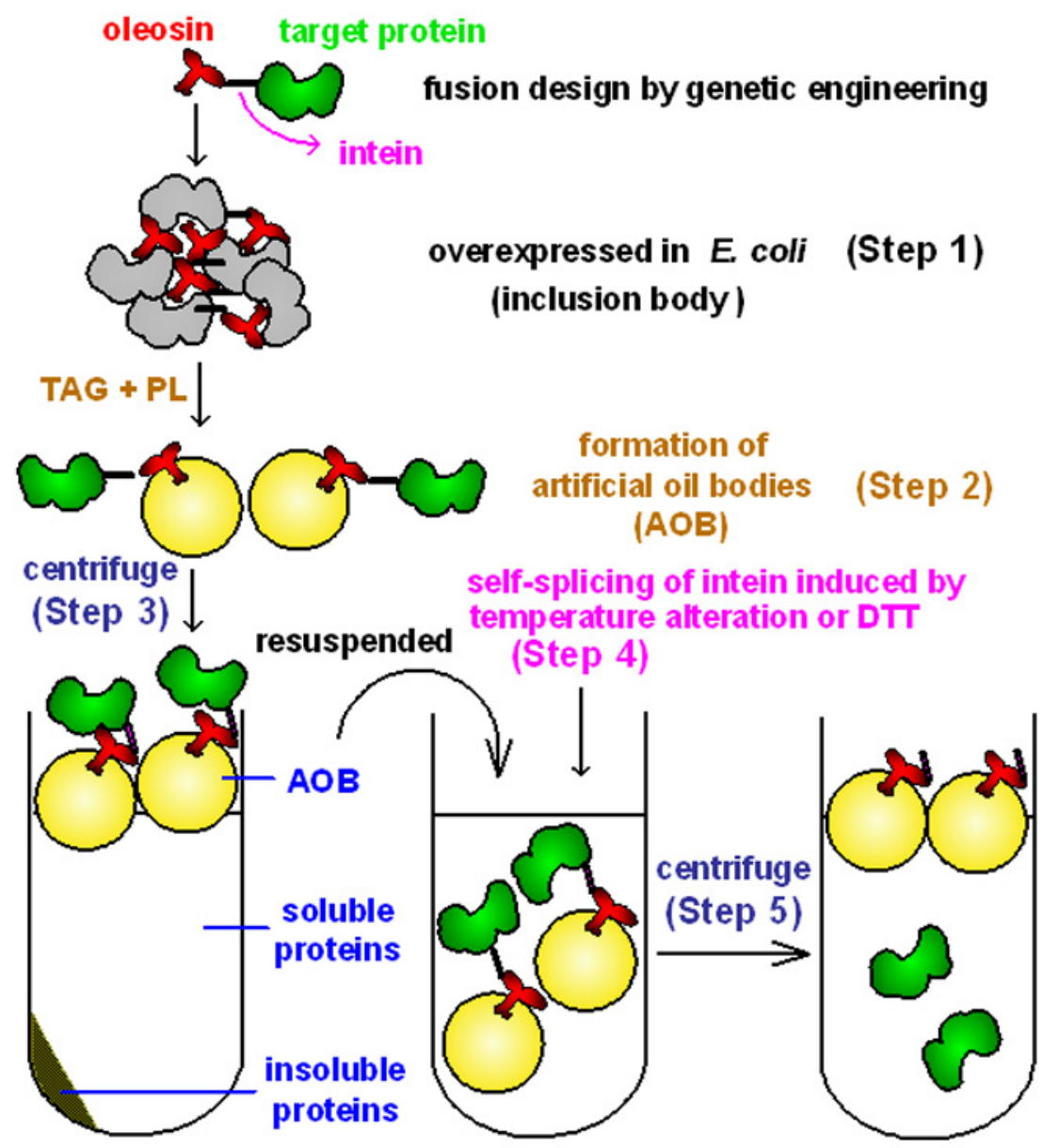

Fig. (3). Schematic representation of the artificial oil body (AOB) expression/purification system. Step 1: over-expression of a target protein in an insoluble oleosin-intein fused form in E. coli. Step 2: constitution of AOBs with the insoluble oleosin-intein fused protein, purified triacylglycerol (TAG) and phospholipid (PL). Step 3: collection of AOBs by centrifugation. Step 4: separation of the target protein from oleosin-intein fused protein in AOBs by temperature alteration or adding dithiothreitol (DTT). Step 5: harvesting the target protein from the ultimate supernatant after centrifugation.

and/or fused bioactive peptides in both dermal and oral applications.

- reducing the number of $\mathrm{N}$-termini available for the initiation of amino peptidase degradation in the rumen. This would enable OBs to remain intact, protecting the encapsulated TAGs from saturation by biohydrogenation in the rumen, and allowing the TAGs to be absorbed in the intestine and incorporated as unsaturated fats.

- anchoring the fused peptides to the OBs more tightly than single oleosin fusions and allows for the purification of individual and multiple fused peptides.

- generating a delivery mechanism for ligand binding fused peptides (e.g., Nanobodies ${ }^{\circledR}$ ) that is effectively a multivalent macromolecule capable of binding to multiple ligands simultaneously.

Up to six tandem repeats of oleosin were successfully expressed both in planta and E. coli; the polyoleosins accumulated in native plant OBs and prokaryotic inclusion bodies. Native OBs and AOBs containing polyoleosins showed increasing thermal stability of the oil body emulsion with an increasing number of oleosin repeats.

\section{CONCLUSIONS}

The properties of oleosins has enabled a broad range of potential applications to be developed; it is likely that more will evolve as the current applications are explored further and as new targets for purification and delivery are married to this technology. A rapid and cost effective first evaluation of their commercial potential has been greatly aided by the development of AOBs. However, the commercial realization of any of these will likely depend on a range of other factors unrelated to the unique characteristics of the protein, in particular, the cost comparison with other recombinant protein expression systems and emulsifiers, and potential allergenicity.

\section{ACKNOWLEDGEMENTS}

The authors gratefully acknowledge Professor Peter Shewry (Rothamsted Research, Harpenden, Herts, UK) for the use of his $\alpha$ helix hairpin model shown in Fig. (1F).

\section{ABBREVIATIONS}

AOBs $=$ Artificial oil bodies 


$$
\begin{aligned}
\text { OBs } & =\text { Oil bodies } \\
\text { TAG } & =\text { Triacylglycerol }
\end{aligned}
$$

\section{REFERENCES}

[1] Tzen JTC, Lie GC, Huang AHC. Characterization of the charged components and their topology on the surface of plant seed oil bodies. J Biol Chem 1992; 267: 15626-34.

[2] Tzen JTC, Wang MMC, Tai SSK, et al. The abundant proteins in sesame seed: storage proteins in protein bodies and oleosins in oil bodies. Adv Plant Physiol 2003; 6: 93-104

[3] Huang AHC. Oleosins and oil bodies in seeds and other organs. Plant Physiol 1996; 110: 1055-61

[4] Jiang PL, Wang CS, Hsu CM, et al. Stable oil bodies sheltered by a unique oleosin in lily pollen. Plant Cell Physiol 2007; 48: 812-21.

[5] Mayfield JA, Fiebig A, Johnstone SE, Preuss D. Gene families from the Arabidopsis thaliana pollen coat proteome. Science 2001; 292: 2482-5.

[6] Lee K, Bih FY, Learn GH, et al. .Oleosins in the gametophytes of Pinus and Brassica and their phylogenetic relationship with those in the sporophytes of various species. Planta 1994; 193: 461-9.

[7] Huang AHC. Oil bodies and oleosins in seeds. Annu Rev Plant Physiol Plant Mol Biol 1992; 43: 177-200.

[8] Loer DS, Herman EM. Cotranslational integration of soybean (Glycine max) oil body membrane protein oleosin into microsomal membranes. Plant Physiol 1993; 101: 993-8.

[9] Murphy DJ. Structure, function and biogenesis of storage lipid bodies and oleosins in plants. Prog Lipid Res 1993; 32: 247-80.

[10] Frandsen GI, Mundy J, Tzen JTC. Oil bodies and their associated proteins, oleosin and caleosin. Physiol Plant 2001; 112: 301-7.

[11] Li M, Smith LJ, Clark DC, et al. Secondary structures of a new class of lipid body proteins from oilseeds. J Biol Chem 1992; 267: 8245-53.

[12] Li M, Murphy DJ, Lee KHK, et al. Purification and structural characterization of the central hydrophobic domain of oleosin. $\mathrm{J}$ Biol Chem 2002; 277: 37888-95.

[13] Lacey DJ, Wellner N, Beaudoin F, et al. Secondary structure of oleosins in oil bodies isolated from seeds of safflower (Carthamus tinctorius L.) and sunflower (Helianthus annuus L.). Biochem J 1998; 334 (Pt 2): 469-77.

[14] Alexander LG, Sessions RB, Clarke AR, et al. Characterization and modelling of the hydrophobic domain of a sunflower oleosin. Planta 2002; 214: 546-51

[15] Ting JT, Lee K, Ratnayake C, et al. Oleosin genes in maize kernels having diverse oil contents are constitutively expressed independent of oil contents. Size and shape of intracellular oil bodies are determined by the oleosins/oils ratio. Planta 1996; 199: 158-65.

[16] Siloto RMP, Findlay K, Lopez-Villalobos A, et al. The accumulation of oleosins determines the size of seed oilbodies in Arabidopsis. Plant Cell 2006; 18: 1961-74.

[17] Murphy DJ. The biogenesis and functions of lipid bodies in animals, plants and microorganisms. Review. Prog Lipid Res 2001; 40: 325-438.

[18] Ting JTL, Balsamo RA, Tarnayake C, et al. Oleosin of plant seed oil bodies is correctly targeted to the lipid bodies in transformed yeast. J Biol Chem 1997; 272: 3699-3706.

[19] Beaudoin F, Wilkinson BM, Stirling CJ, Napier JA. In vivo targeting of a sunflower oil body protein in yeast secretory (sec) mutants. Plant J 2000; 23: 159-70.

[20] Lin LJ, Liao PC, Yang HH, Tzen JTC. Determination and analyses of the N-termini of oil-body proteins, steroleosin, caleosin and oleosin. Plant Physiol Biochem 2005; 43: 770-6.

[21] Abell BM, Holbrook LA, Abenes M, et al. Role of the proline knot motif in oleosin endoplasmic reticulum topology and oil body targeting. Plant Cell 1997; 9: 1481-93.

[22] Sarmiento C, Ross JHE, Herman E, Murphy DJ. Expression and subcellular targeting of a soybean oleosin in transgenic rapeseed. Implications for the mechanism of oil-body formation in seeds. Plant J 1997; 11: 783-96.

[23] Abell BM, Hahn M, Holbrook LA, Moloney MM. Membrane topology and sequence requirements for oil body targeting of oleosin. Plant J 2004; 37: 461-70.
Lin LJ, Tzen JTC. Two distinct steroleosins are present in seed oil bodies. Plant Physiol Biochem 2004; 42: 601-8.

Peng CC, Lin IP, Lin CK, Tzen JTC. Stability enhancement of native and artificial oil bodies by genipin crosslink. Taiwan patent I250466. 2006 Mar.

[26] Pons L, Cherry C, Romano A, et al. The $18 \mathrm{kDA}$ peanut oleosin is a candidate allergen for IgE-mediated reactions to peanuts. Allergy 2002; 57(Suppl 72): 88-93.

[27] Akkerdaas JH, Schocker F, Vieths S, et al. Cloning of oleosin, a putative new hazelnut allergen, using a hazelnut cDNA library. Mol Nutr Food Res 2006; 50: 18-23.

[28] Leduc V, Moneret-Vautrin DA, Tzen JTC, et al. Identification of oleosins as major allergens in sesame seed allergic patients. Allergy 2006; 349-56.

[29] Moloney MM. Sembiosys Genetics Inc. Oil-body proteins as carriers of high-value peptides in plants. United States patent US 659835. $1991 \mathrm{Feb}$

[30] Moloney MM, van Rooijen G. Sembiosys Genetics Inc. Expression of epidermal growth factor in plant seeds. United States patent US 7091401. 2006 Aug.

[31] Moloney MM. Sembiosys Genetics Inc. Oil-body proteins as carriers of high-value peptides in plants. United States patent US 5650554. $1997 \mathrm{Jul}$

[32] Deckers HM, van Rooijen G, Boothe J, et al. Sembiosys Genetics Inc. Products for topical applications comprising oil bodies. United States patent US 6582710. 2003 Jun.

[33] Moloney MM, Boothe J, van Rooijen G. Sembiosys Genetics Inc. Oil bodies and associated proteins as affinity matrices. United States patent US 6509453. 2003 Jan.

[34] Szarka S, van Rooijen G, Moloney MM. Sembiosys Genetics Inc. Methods for the production of multimeric immunoglobulins, and related compositions. United States patent US 7098383. 2006 Aug.

[35] van Rooijen G, Zaplachinski S, Heifetz PB, et al. Sembiosys Genetics, Inc. Methods for the production of multimeric protein complexes, and related compositions. United States patent US 20060179514. 2006 Oct.

[36] Cheng KJ, Selinger LB, Liu JH, et al. Her Majesty the Queen in right of Canada. Xylanase obtained from an anaerobic fungus. United States patent US 6137032. 2000 Oct.

[37] Hou RCW, Lin MY, Wang MMC, Tzen JTC. Increase of viability of entrapped cells of Lactobacillus delbrueckii ssp. bulgaricus in artificial sesame oil emulsions. J Dairy Sci 2003; 86: 424-8.

[38] Harada T, Kashihara K, Nio N. Food Research \& Development Laboratories, Ajinomoto Co., Inc. OLEOSIN/PHOSPHOLIPID COMPLEX AND PROCESS FOR PRODUCING THE SAME. World Patent WO/2002/026788. 2002 Feb.

[39] Deckers HM, van Rooijen G, Boothe J, et al. Sembiosys Genetics Inc. Uses of oil bodies. United States patent US 6210742. 2001 Apr.

[40] Berry MJ, Cox AR, Keenan RD, Quail PJ. Good Humor-Breyers Ice Cream, Division of Conopco, Inc Ice confection and its manufacturing process. United States patent US 20050037111. 2005 Feb.

[41] McCarthy J, Nestec S.A. Recombinant oleosins from cacao and their use as flavoring or emulsifying agents. United States patent US 7126042. 2006 Oct.

[42] Peng CC, Chen JCF, Shyu DJH, et al. A system for purification of recombinant proteins in Escherichia coli via artificial oil bodies constituted with their oleosin-fused polypeptides. J Biotechnol 2004; 111: 51-7.

[43] Capuano F, Beaudoin F, Napier JA, Shewry PR. Properties and exploitation of oleosins. Biotechnol Adv 2007; 25: 203-6.

[44] Peng CC, Lin IP, Lin CK, Tzen JTC. Size and stability of reconstituted sesame oil bodies. Biotechnol Prog 2003; 19: 1623-6.

[45] Roux E, Baumberger S, Axelos MA, Chardot T. Oleosins of Arabidopsis thaliana: expression in Escherichia coli, purification, and functional properties. J Agric Food Chem 2004; 52: 5245-9.

[46] Peng CC, Shyu DJH, Chou WM, et al. Method for bacterial expression and purification of sesame cystatin via artificial oil bodies. J Agric Food Chem 2004; 52: 3115-9.

[47] Chiang CJ, Chen HC, Chao YP, Tzen JTC. Efficient system of artificial oil bodies for functional expression and purification of recombinant nattokinase in Escherichia coli. J Agric Food Chem 2005; 53: 4799-804. 
[48] Chiang CJ, Chen HC, Chao YP, Tzen JTC. One-step purification of insoluble hydantoinase overproduced in Escherichia coli. Protein Expr Purif 2007; 52: 14-8.
[49] Scott RW, Arcus VL, Roberts NJ. Agriculture Victoria Services Pty Ltd. Polyoleosins. World patent WO/2007/045019. 2007 Apr. 